\title{
EXPLOTACIÓN DE LOS YACIMIENTOS DE FOSFATOS EN BAYÓVAR
}

\author{
PHOSPHATE DEPOSITS OF BAYOVAR EXPLOITATION
}

\author{
Juvenal Cabezas Oruna ${ }^{1}$
}

\section{RESUMEN}

Los extensos yacimientos de fosfatos ubicados en Bayóvar fueron descubiertos en la década de 1950, en la costa del departamento de Piura. Más de medio siglo después, a mediados del año 2010, se inició su explotación a cargo de la empresa Miski Mayo, subsidiaria de la brasileña Vale, que ganó un concurso público internacional llevado a cabo por PROINVERSIÓN en el año 2005. El presente artículo describe el área de Bayóvar, así como las características del yacimiento; explica también el desarrollo del proyecto que llevó adelante Vale para la extracción y procesamiento de la roca fosfórica con el objetivo de obtener un concentrado de fosfatos con una concentración mínima de $29 \%$ de $\mathrm{P}_{2} \mathrm{O}_{5}$ y una capacidad de procesamiento de 3.9 millones de toneladas anuales, mediante una inversión de 566 millones de US\$. Se describe, asimismo, en forma resumida, los nueve componentes de la infraestructura total: mina a cielo abierto, planta concentradora, carretera industrial, zona de descarga de camiones, faja transportadora, zona de secado y almacenamiento, puerto, sistema de agua de mar, y línea de transmisión eléctrica. Se revisan también las inversiones efectuadas por Vale, así como nuevos proyectos en la zona a cargo de otras empresas. Se presentan luego las estadísticas de producción de fosfatos desde el 2010 y se plantea que un sector de industrialización en el Perú sea la producción de fertilizantes, teniendo como materia prima los fosfatos de Bayóvar, discutiéndose algunos procesos de transformación de los fosfatos.

Palabras clave: fosfatos, Bayóvar, roca fosfórica, yacimiento, mina, concentración.

\begin{abstract}
The extensive phosphate deposits of Bayovar were discovered in the 1950 decade in the coast of Piura. After more than half a century, the company Miski Mayo, a subsidiary of Vale, which won an international public tender called by PROINVERSION in 2005, began the exploitation of this area in the middle of 2010. This article describes the Bayovar area as well as the deposit characteristics, and also the development of the project undertaken by Vale for the extraction and processing of phosphate rock in order to obtain a phosphate concentrate with a minimum concentration of $29 \%$ of $\mathrm{P}_{2} \mathrm{O}_{5}$ and a processing capacity of 3.9 millions of tons each year, with an investment of US\$ 566 millions. It also describes, in summary, the nine components of the total infrastructure: open pit mine, concentrator plant, industrial road, truck unloading area, conveyor, drying and storage area, port, sea water system, and electric transmission line. We review as well, the investments made by Vale and new projects in the area of Bayovar developed by other companies. It then presents the phosphate production statistics from 2010 and considers that a sector of industrialization in Peru would be the production of fertilizers, having the Bayovar phosphates as raw materials, discussing also some processes for the transformation of phosphates.
\end{abstract}

Keywords: Phosphates, Bayovar, phosphate rock, deposits, mine, concentration.

1 Ingeniero Químico, Máster en Administración, Doctor en Medioambiente y Desarrollo Sostenible, profesor principal de la Universidad Ricardo Palma, Av. Benavides 5440, Lima, Perú. <juvecabezas@yahoo.com> 


\section{INTRODUCCIÓN}

Después de medio siglo de haber sido descubiertos, en agosto de 2010 empezó la exportación de los fosfatos de Bayóvar al Brasil por intermedio de la empresa Miski Mayo S.R.L., subsidiaria de la empresa brasileña Vale do Río Doce. Para la explotación y exportación de los fosfatos, Vale desarrolló el Proyecto Bayóvar, el cual tiene un enfoque minero no metálico que contempló la explotación de un yacimiento superficial de roca fosfórica, el procesamiento de la misma para producir concentrados y su embarque para exportación.

Los yacimientos de fosfatos de Bayóvar están ubicados al sur de la bahía de Sechura, en la costa del departamento de Piura. La riqueza del subsuelo de esta zona fue detectada en la década de 1950 y desde entonces fue extensamente explorada y estudiada por empresas estatales y privadas, nacionales y extranjeras, quienes determinaron que se trataba de un yacimiento gigante. Mis primeras experiencias con Bayóvar se remontan a la década de 1970 cuando visité la zona por primera vez con el grupo de trabajo del proyecto 'Refinería de Exportación de Bayóvar' que había formado PETROPERÚ para refinar el petróleo que se había descubierto en 1971 en la selva norte del departamento de Loreto con el famoso pozo petrolero 'Trompeteros' y también para exportar los combustibles que se iban a obtener en dicha refinería. La zona tiene tres ecosistemas: el desierto ubicado al sur de Bayóvar, los bosques secos ubicados al este y el gran cerro Illescas al oeste, cuya parte más baja, ubicada en la zona sur de la bahía de Sechura, iba a ser utilizada también como punto final del Oleoducto Norperuano y puerto de exportación de petróleo. Con el tiempo, el proyecto de la refinería de exportación no se concretó, pero sí se construyó el oleoducto, que ha permitido, hasta el día de hoy, llevar el petróleo de la selva de Loreto a la costa de Piura, y exportarlo por el puerto petrolero de Bayóvar.

\section{EL PROYECTO}

La empresa Vale se adjudicó el Proyecto Bayóvar a través de un concurso público internacional llevado a cabo por PROINVERSIÓN en el año 2005, por el cual obtuvo la concesión del área "Bayóvar 2" para explotarla durante 27 años (El Comercio, 2005). El proyecto inicial se concluyó en 5 años y las operaciones de Bayóvar empezaron oficialmente a mediados del 2010.

El grupo Vale do Río Doce del Brasil es uno de los conglomerados mineros diversificados más grandes del mundo. Está presente en 30 países y es uno de los mayores productores mundiales de minerales ferrosos y pellets. Por ejemplo, en el 2009 las inversiones del grupo en el negocio de minerales no ferrosos se concentraron en los proyectos Onca Puma de níquel (Brasil), Goro de níquel (Nueva Caledonia), Salobo de cobre (Brasil), Tres Valles de cobre (Chile) y Bayóvar de concentrados de fosfatos en Perú (Revista Mercado Energía, 2009).

\section{UBICACIÓN}

Estos yacimientos se ubican en el distrito y provincia de Sechura, departamento de Piura, en la zona denominada Bayóvar, al sur de la bahía de Sechura, aproximadamente a 1000 kilómetros al norte de Lima, a 110 kilómetros al sur de la ciudad de Piura y a 30 kilómetros del Océano Pacífico.

El acceso al área de operaciones está ubicado en el kilómetro 900 de la carretera Panamericana Norte, entre las ciudades de Mórrope y Piura. En este punto, conocido como Pampa de Michales, se toma el desvío hacia el noroeste que corresponde a la antigua carretera Bayóvar-Chiclayo y se avanza cerca de 50 kilómetros hasta llegar a la bahía de Sechura, en donde PETROPERÚ tiene las operacio- 


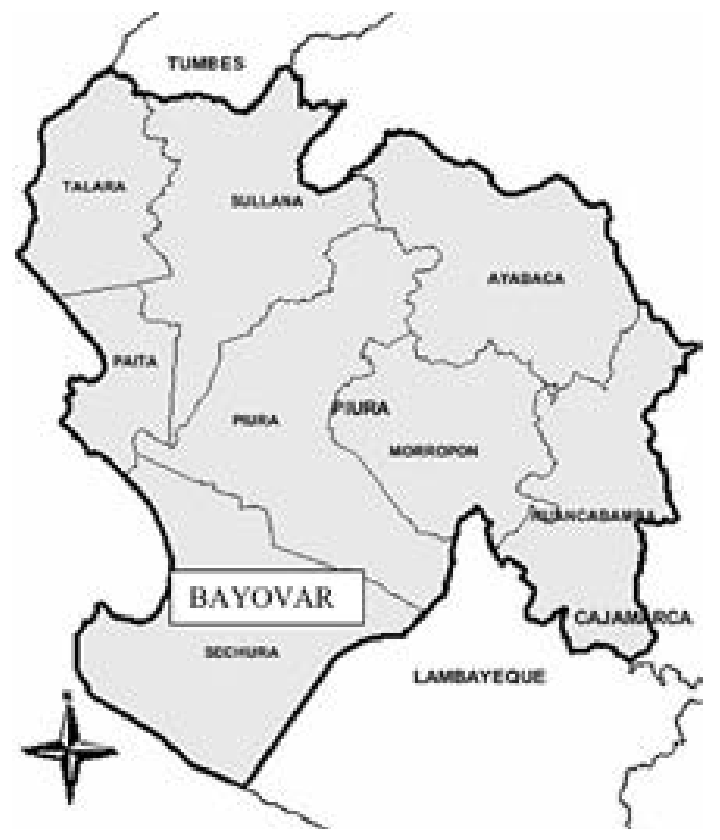

Figura 1: Departamento de Piura nes portuarias para la exportación del petróleo de la selva norte. Para ir a la mina, es necesario tomar un desvío hacia el oeste en la carretera de 50 kilómetros mencionada anteriormente.

\section{RESERVAS}

El yacimiento de Bayóvar es uno de los más grandes de Sudamérica. Está compuesto por varias capas de roca fosfórica de unos 2 metros de espesor a la que se le llama "mineral”; dichas capas están intercaladas con diatomitas. La revista Infraestructura Peruana (2011) indica que, sólo en sus cinco primeras capas, el yacimiento tiene una reserva de 238 millones de toneladas de roca fosfórica.

Este mineral se formó durante millones de ańos debido los sedimentos de origen vegetal y animales marinos, es decir, algas y peces propios del mar que existía

en este lugar hace millones de años. La roca fosfórica solo está cubierta por la arena y por material sedimentario, propio de las ecorregiones del desierto del pacífico y bosque seco ecuatorial, por lo que su extracción es relativamente fácil y bastante económica. Debido a las características del mineral, no hay necesidad de efectuar perforaciones y voladuras, estimándose que la explotación irá hasta una profundidad de 50 metros.

\section{CARACTERÍSTICAS PRINCIPALES}

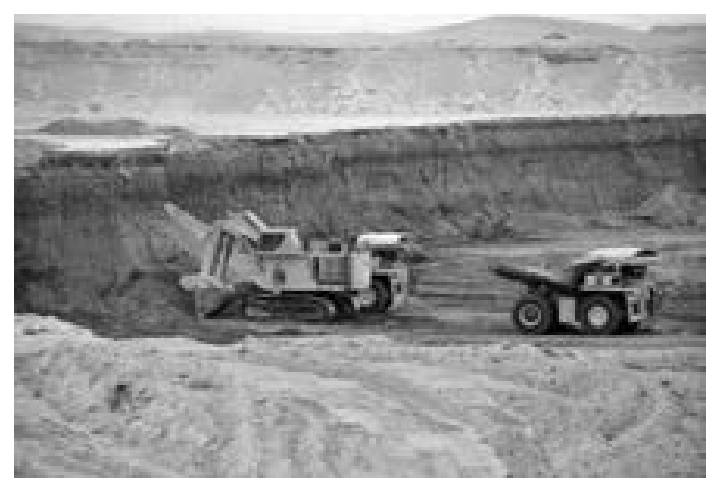

Figura 2: Extracción de la roca fosfórica

El objetivo de la operación es la extracción y procesamiento de la roca fosfórica para obtener un producto con una concentración mínima de $29 \%$ de $\mathrm{P}_{2} \mathrm{O}_{5}$ para su posterior exportación a los mercados internacionales y especialmente al Brasil. Según el estudio de Impacto Ambiental (Golder, 2007), la infraestructura total del proyecto Fosfatos de Bayóvar comprende 9 componentes que se reseñan brevemente: (1) Mina a cielo abierto, (2) planta concentradora, (3) carretera industrial, (4) zona de descarga de camiones, (5) faja transportadora, (6) zona de secado y almacenamiento, (7) puerto, (8) sistema de agua de mar, y (9) línea de transmisión eléctrica.

\section{Mina a cielo abierto}

La operación se inicia en la mina a tajo abierto que tiene un área de 4200 hectáreas. Las características del yacimiento permiten que el minado se realice mediante excavación con palas hidráulicas, buldó- 
ceres, cargadores frontales y camiones. Así, se remueve la arena y el material sedimentario hasta dejar expuesto el mineral (roca fosfórica). Se procede a sacar el mineral y se coloca en una faja transportadora que lo lleva a la planta concentradora.

\section{Planta concentradora}

Tiene una capacidad de casi 4 millones de toneladas anuales. Una vez que se extrae el mineral bruto del suelo es llevado mediante camiones y fajas a la planta concentradora, donde es sometido en forma sucesiva a lavados con agua de mar y separaciones gravimétricas. En la etapa final de esta área, el concentrado es lavado con agua desmineralizada, obtenida del mar mediante ósmosis inversa, para retirarle la mayor cantidad de sales, obteniéndose así concentrados de fosfatos con una humedad promedio de $15 \%$. No se usan productos químicos en esta etapa. En esta zona de la planta concentradora (figura 3) está ubicada también la planta desmineralizadora de agua de mar.

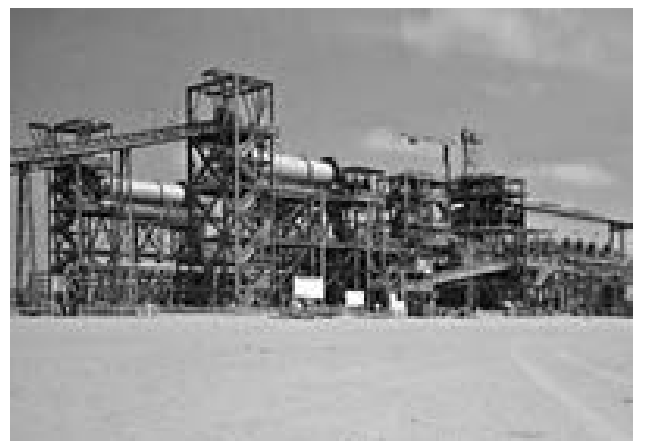

Figura 3: Planta Concentradora

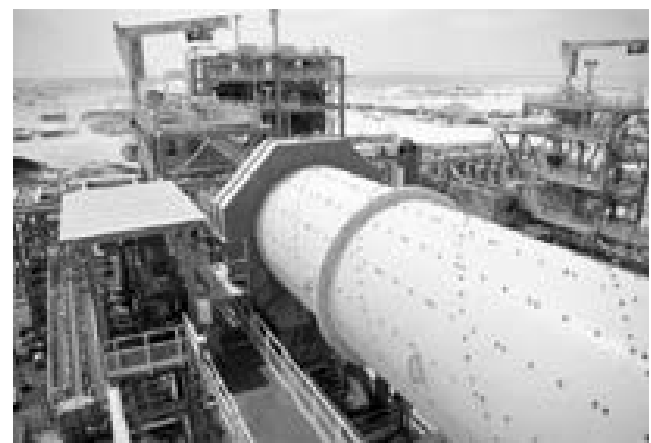

Figura 4: Tambor lavador

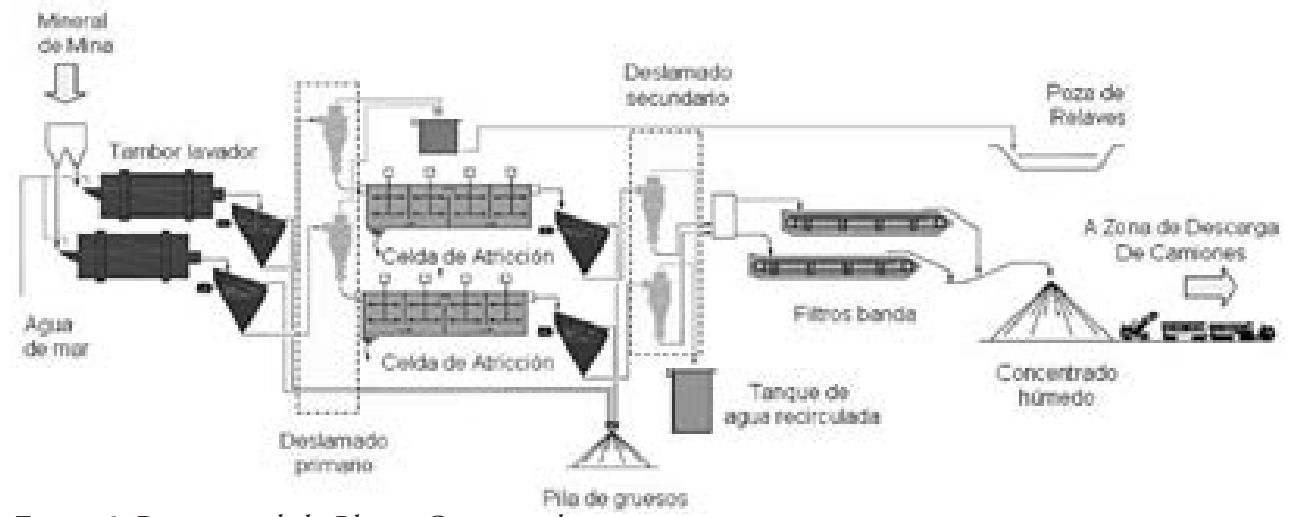

Figura 5: Diagrama de la Planta Concentradora

\section{Carretera industrial}

A continuación, los concentrados de fosfatos, con una humedad de $15 \%$, se llevan a la zona de descarga mediante grandes camiones de 70 toneladas de capacidad que aprovechan una carretera de 30 kilómetros de largo.

\section{Zona de descarga de camiones}

Está ubicada en la zona portuaria, al sur de la bahía de Sechura, y consiste de 2 grandes tolvas de recepción de concentrados. 


\section{Faja transportadora}

Tiene una longitud de 5 kilómetros. Los camiones descargan en ella y lleva los concentrados hasta la zona de secado y almacenamiento.

\section{Zona de secado y almacenamiento}

Está ubicada al sur de la bahía de Sechura, en la zona baja del cerro Illescas. En este lugar, los concentrados de fosfatos, que tienen una humedad promedio de 15\%, son secados en 2 hornos rotatorios a gas natural hasta una humedad promedio de $3 \%$ y luego son almacenados en un silo. De aquí, los concentrados de fosfatos son llevados hasta el puerto mediante una faja transportadora para ser embarcados.

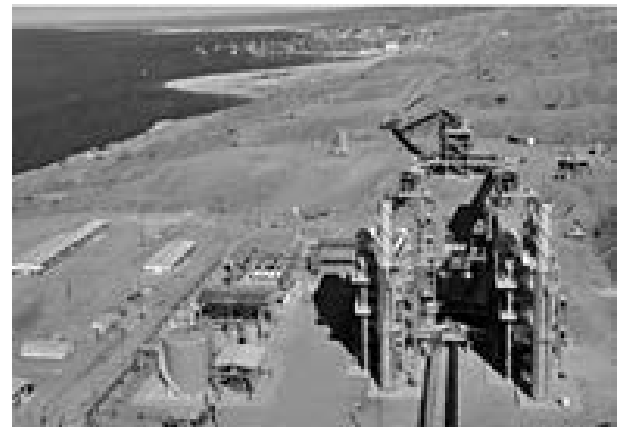

Figura 6: Zona de secado

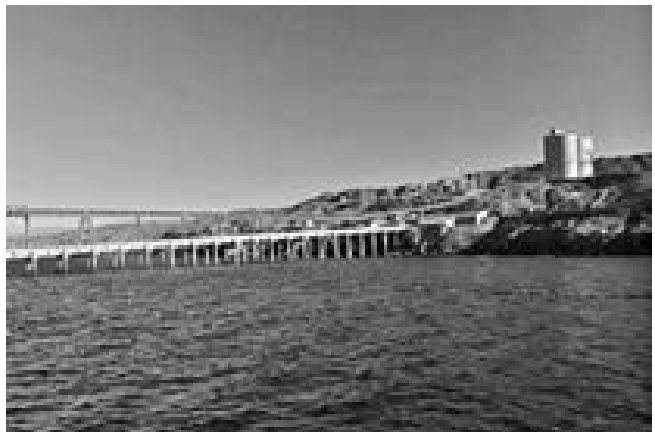

Figura 7: Puerto de Bayóvar

\section{Puerto}

El puerto está ubicado en plena Bahía de Sechura, zona sur, a 7 kilómetros al este del puerto petrolero de PETROPERÚ. Tiene una faja transportadora y una zona de descarga de los concentrados a los buques. El brazo cargador de buques se desplaza en forma paralela al muelle y tiene un tubo o brazo telescópico basculante. El puerto tiene una plataforma principal de 16 metros de ancho y 187 metros de largo, por lo que puede recibir buques de hasta 100 mil toneladas de peso muerto (100,000 DWT).

\section{Sistema de agua de mar}

En la planta concentradora se utilizan grandes volúmenes de agua de mar y agua desmineralizada. El agua de mar se capta en el muelle mediante bombas verticales que la envían hasta la planta concentradora, ubicada a casi 40 kilómetros de distancia, mediante una tubería de 36 pulgadas de diámetro en material HDPE. El agua de mar es descargada en pozas de sedimentación, para separar principalmente la arena, y el agua de rebose va a la poza de almacenamiento para usarse en el lavado del mineral bruto. Una parte del agua de mar que llega a la planta concentradora (500 metros cúbicos por hora) es procesada adicionalmente mediante ósmosis inversa para obtener agua desmineralizada, la cual se usa en el lavado final de los concentrados de fosfatos.

\section{Línea de transmisión eléctrica}

Las operaciones demandan $18 \mathrm{MW}$ de electricidad, la cual es cubierta mediante una línea de 90 kilómetros de extensión, que se deriva del Sistema Interconectado Nacional a la altura del kilómetro 912 de la carretera Panamericana Norte. 


\section{INVERSIONES}

El proyecto inicial está en operación desde el 2010 y tiene una capacidad anual de 3.9 millones de toneladas. Demandó una inversión de US\$ 566 millones. Vale do Rio viene evaluando una ampliación de la planta para elevar en 50\% su producción de roca fosfórica hasta 5.8 millones de toneladas (Revista Panorama Industrial, 2011).

La exitosa operación de Vale ha hecho que ésta y otras empresas exploren nuevas zonas en la costa piurana, encontrándose otras áreas con gran potencial de roca fosfórica. Por este motivo, las siguientes empresas están desarrollando proyectos en la zona:

a) Focus Ventures (Canadá): el proyecto “Bayóvar 12” está terminando la fase de exploración. Iniciaría la explotación de fosfatos a inicios del año 2017.

b) Cementos Pacasmayo SAA / Fosfatos del Pacífico (Perú): el proyecto "Bayóvar 9" se encuentra en fase de pre-inversión.

c) GrowMax Agri Corp (Canadá) /Americas Potash Perú: el proyecto Salmueras de Bayóvar tiene como objetivo la explotación de salmueras subterráneas, mediante evaporación solar del agua y obtención de cloruro de potasio y otras sales industriales.

\section{ESTADÍSTICAS DE PRODUCCIÓN}

Las estadísticas de producción minera no metálica de fosfatos, de acuerdo al Ministerio de Energía y Minas (2010 al 2014, año 2015 aún sin información), son las siguientes:

$\begin{array}{ll}\text { Estrato: } & \text { Régimen especial } \\ \text { Categoría: } & \text { Gran minería } \\ \text { Titular: } & \text { Compañía minera Miski Mayo S.R.L. } \\ \text { Unidad: } & \text { Bayóvar } 2 \\ \text { Producto: } & \text { Fosfatos }\end{array}$

Estadísticas de explotación:

TABLA 1: ESTADÍSTICAS DE EXPLOTACIÓN

\begin{tabular}{|c|c|c|c|c|c|c|}
\hline AÑO & 2010 & 2011 & 2012 & 2013 & 2014 & 2015 \\
\hline Miles de TM & 1134 & 8889 & 10338 & 14832 & 10868 & $\left.{ }^{*}\right)$ \\
\hline
\end{tabular}

(*) Sin información

\section{FOSFATOS E INDUSTRIALIZACIÓN DEL PERÚ}

El empleo de fertilizantes en el Perú y el mundo ha aumentado de manera continua, tanto de los fertilizantes artificiales, como los naturales. Por este motivo, se debe generar una corriente de industrialización en el Perú que aproveche los fosfatos de Bayóvar y que se sume a la ansiada industrialización que se debe iniciar con el uso del gas de Camisea. Las propiedades de los productos químicos derivados de los fosfatos son únicas, debido al importante papel que juega el fósforo en numerosos procesos bioquímicos al interior de personas, animales y plantas, a la capacidad de los polifosfatos para generar moléculas complejas o para secuestrar a muchos cationes metálicos, así como a su flexibilidad para formar varios tipos de polímeros orgánicos e inorgánicos. 
Austin (1988) señala que las rocas fosfóricas son esencialmente fluoropatita mezclada con diferentes proporciones de otros compuestos de calcio, flúor, hierro, aluminio y silicio. La fórmula de la fluoropatita es $\mathrm{CaF}_{2} \cdot 3 \mathrm{Ca}_{3}\left(\mathrm{PO}_{4}\right)_{2}$. Este compuesto es extremadamente insoluble. Los diferentes procesos químicos que se usan para hacer que el contenido de $\mathrm{P}_{2} \mathrm{O}_{5}$ sea más soluble, especialmente para su absorción por las plantas y su presencia en los jugos de las plantas, consisten en la manufactura de varios superfosfatos y en la desfluorización de la fluoropatita por calcinación a las temperaturas de fusión incipiente de 1400 a $1500^{\circ} \mathrm{C}$ con sílice o con ácido fosfórico.

Fabricación de superfosfatos: El método más importante para producir fosfatos para fertilizantes es la acidulación de la roca fosfórica. La siguiente es una expresión más probable de la reacción principal:

$$
\begin{array}{ccc}
\mathrm{CaF}_{2} \cdot 3 \mathrm{Ca}_{3}\left(\mathrm{PO}_{4}\right)_{2}+7 \mathrm{H}_{2} \mathrm{SO}_{4}+3 \mathrm{H}_{2} \mathrm{O} & 3 \mathrm{CaH}_{4}\left(\mathrm{PO}_{4}\right)_{2} \cdot \mathrm{H}_{2} \mathrm{O}+2 \mathrm{HF}+7 \mathrm{CaSO}_{4} \\
\text { Roca fosfórica } & 53-57^{\circ} \text { Bé } & \text { Fosfato monocálcico }
\end{array}
$$

Se tiene como resultado un superfosfato con 16 a 20\% de $\mathrm{P}_{2} \mathrm{O}_{5}$ disponible. La manufactura del superfosfato incluye cuatro pasos: (1) preparación de la roca fosfórica, (2) mezclado con ácido, (3) curado y secado de la lechada original al completar las reacciones y (4) molienda y empacado del producto terminado. En todas las plantas se pulveriza primero la roca hasta que el $75 \%$ pasa a través de un tamiz de 200 mallas para obtener una velocidad de reacción mayor, donde se utiliza con más eficiencia el ácido sulfúrico, por lo que se requiere menos ácido y se obtiene un producto de grado superior en mejores condiciones.

Elaboración de superfosfato normal: El proceso se inicia con la molienda de la roca fosfórica (90\% inferior a la malla 100). Luego se mezcla con ácido sulfúrico $51^{\circ}$ Bé. El calor de dilución calienta

\begin{tabular}{|c|c|c|c|}
\hline Proceso & Materias primas y reactivos & Productos principales y derivados & Subproductos \\
\hline Acidulación & $\begin{array}{l}\text { Roca fosfórica, } \\
\text { ácido sulfúrico, } \\
\text { ácido nítrico, } \\
\text { ácido fosfórico, } \\
\text { ácido clorhídrico, } \\
\text { amoniaco y } \\
\text { cloruro de potasio. }\end{array}$ & $\begin{array}{l}\text { Superfosfato, } \\
\text { ácido fosfórico (proceso húmedo), } \\
\text { superfosfato triple, } \\
\text { fosfato monoamónico, } \\
\text { fosfato diamónico y } \\
\text { fosfato monopotásico. }\end{array}$ & $\begin{array}{l}\text { Compuestos de flúor, } \\
\text { Vanadio y } \\
\text { Uranio (limitado). }\end{array}$ \\
\hline $\begin{array}{l}\text { Reducción de horno } \\
\text { eléctrico }\end{array}$ & $\begin{array}{l}\text { Roca fosfórica, } \\
\text { fundente de sílice, } \\
\text { coque (para reducción), } \\
\text { energía eléctrica y agua de } \\
\text { condensación. }\end{array}$ & $\begin{array}{l}\text { Fósforo, } \\
\text { ácido fosfórico, } \\
\text { superfosfato triple, } \\
\text { varias sales de } \mathrm{Na}, \mathrm{K}, \mathrm{NH} 4 \text { y Ca, } \\
\text { pentaóxido de fósforo y haluros. }\end{array}$ & $\begin{array}{l}\text { Compuestos de flúor, } \\
\text { monóxido (CO), } \\
\text { escoria (para agregado de lastre } \\
\text { de ferrocarril, rellenos), } \\
\text { ferrofósforo y } \\
\text { Vanadio. }\end{array}$ \\
\hline $\begin{array}{l}\text { Metafosfato de } \\
\text { calcio }\end{array}$ & $\begin{array}{l}\text { Roca fosfórica, } \\
\text { fósforo, } \\
\text { aire u oxígeno y combustible. }\end{array}$ & Metafosfato de calcio & Compuestos de flúor \\
\hline $\begin{array}{l}\text { Calcinación } 0 \\
\text { desfluorización }\end{array}$ & $\begin{array}{l}\text { Roca fosfórica, } \\
\text { sílice, } \\
\text { agua o vapor y combustible. }\end{array}$ & Fosfato desfluorizado & Compuestos de flúor \\
\hline
\end{tabular}

TABLA 2: PROCESAMIENTO, PRODUCTOS Y SUBPRODUCTOS DE ROCA FOSFÓRICA 
el ácido sulfúrico hasta la temperatura apropiada para la reacción y el exceso de calor se disipa por evaporación del agua extra que se ha añadido. El curado, o finalización de la reacción química, requiere de 4 a 6 semanas para alcanzar una disponibilidad aceptable de $\mathrm{P}_{2} \mathrm{O}_{5}$ para alimento de las plantas.

Elaboración de superfosfato triple: Este fertilizante es mucho más concentrado que el superfosfato común, pues contiene entre 45 y $46 \%$ de $\mathrm{P}_{2} \mathrm{O}_{5}$ disponible. Se prepara por la acción del ácido fosfórico sobre la roca fosfórica:

$$
\begin{gathered}
\mathrm{CaF}_{2} \cdot 3 \mathrm{Ca}_{3}\left(\mathrm{PO}_{4}\right)_{2}+14 \mathrm{H}_{3} \mathrm{PO}_{4}= \\
\text { Roca fosfórica }
\end{gathered}
$$

El proceso empieza con la molienda de la roca fosfórica. Luego, la roca se mezcla con ácido fosfórico en un reactor de dos etapas. La lechada resultante se rocía dentro del granulador. El producto de este se seca y se tamiza; el material más grande se tritura y se vuelve a enfriar. El producto final se transporta hacia el lugar de almacenamiento, en donde se cura durante 4 a 6 semanas, tiempo en el que ocurre una mayor reacción entre el ácido y la roca, lo que aumenta la disponibilidad de $\mathrm{P}_{2} \mathrm{O}_{5}$ como alimento para las plantas.

\section{CONCLUSIONES}

- Los inmensos yacimientos de fosfatos de Bayóvar se están explotando desde el 2010 pero se están exportando en su totalidad. Constituyen recursos no renovables que pueden usarse como fertilizantes de aplicación directa en nuestro país, ya que una de las ventajas de este producto es su solubilidad, lo cual ayuda a que las plantas absorban rápidamente el mineral en forma de ion fosfato P2O5.

- Así también, los fosfatos de Bayóvar pueden servir como materia prima para la producción de ácido fosfórico, que sirve para fabricar una familia de fertilizantes muy importante a nivel mundial que incluye al fosfato monoamónico (MAP), fosfato di-amónico (DAP), superfosfato triple (TSP) y superfosfato simple (SSP). Estos fertilizantes, junto con la urea, el nitrato de amonio, el sulfato de amonio y el cloruro de potasio, constituyen la gran familia de fertilizantes NPK, muy requeridos como abonos en los campos agrícolas, debido a que contienen nitrógeno, fósforo y potasio.

- En adición a la obtención de fertilizantes, una transformación de los fosfatos obtenidos de la roca fosfórica lleva a la obtención de numerosos productos como es el caso de plastificantes, rellenos de detergentes, ablandadores de agua, insecticidas, aditivos de productos petroleros y metalúrgicos, etc.

- Todas estas aplicaciones llevan a proponer que una parte de dichos fosfatos se queden en el país para su transformación y para contribuir a la industrialización del Perú, de manera similar a como se quiere industrializar el gas de Camisea. Campodónico (2015) señala que:

"Desde el 2005 la importación de fertilizantes ha crecido enormemente, tanto por la expansión de la producción para el mercado interno, como por las exportaciones no tradicionales (espárrago, mango, cebolla, uvas, entre otros). Asi, en el 2012 la importación de todos los tipos de fertilizantes (urea, fosfato diamónico, nitrato de amonio, sulfato de amonio) llegó a 900 mil toneladas por un valor de US\$ 400 millones. Y van a seguir creciendo porque se están ampliando las áreas de cultivo a partir de nuevos proyectos de irrigación y la ampliación de los existentes (Olmos, Chavimochic, Majes-Sihuas, Chira-Piura, entre otros)". 


\section{REFERENCIAS}

Austin, G. (1988). Manual de Procesos Químicos en la Industria. México: McGraw-Hill, 315-324.

Campodónico, H. (13 de julio de 2015). Bayóvar, roca fosfórica y diversificación productiva. La República, Lima.

Exportarán 33 mil toneladas de fosfatos al Brasil desde Bayóvar. (27 de julio de 2010). El Comercio, Lima.

Golder Associates Perú S.A. (noviembre, 2007). Estudio de Impacto Ambiental - Proyecto de Fosfatos Bayóvar. Piura, Perú. Resumen ejecutivo. Lima.

Ministerio de Energía y Minas (enero-diciembre, 2010-2015) Estadísticas de Producción de Minerales No Metálicos. Lima.

Tras larga espera se subastan fosfatos de Bayóvar. (13 de marzo de 2005). El Comercio, Lima.

Vale, la Empresa (agosto, 2009). Revista Mercado Energía.

Vale aprobaría ampliación de planta de fosfatos de Bayóvar a fines de 2011 (setiembre, 2011). Revista Panorama Industrial.

Vega Farfán, José (noviembre, 2009). Vale, Mina de Fosfatos Bayóvar. Piura.

Yacimiento de Fosfatos de Bayóvar (setiembre, 2011). Revista Infraestructura Peruana.

www.focusventuresltd.com

www.cementospacasmayo.com.pe

www.growmaxagricorp.com 